\title{
Neutral endopeptidase inhibits prostate cancer cell migration by blocking focal adhesion kinase signaling
}

\author{
Makoto Sumitomo, ${ }^{1}$ Ruoqian Shen, ${ }^{1}$ Marc Walburg, ${ }^{2}$ Jie Dai, ${ }^{1}$ Yiping Geng, ${ }^{1}$ \\ Daniel Navarro, ${ }^{1}$ Guy Boileau, ${ }^{3}$ Christos N. Papandreou, ${ }^{4}$ Filippo G. Giancotti, ${ }^{5}$ \\ Beatrice Knudsen, ${ }^{2}$ and David M. Nanus ${ }^{1,6}$ \\ ${ }^{1}$ Urologic Oncology Research Laboratory, Department of Urology, and \\ ${ }^{2}$ Department of Pathology, Weill Medical College of Cornell University, New York, New York, USA \\ ${ }^{3}$ Department of Biochemistry, Faculty of Medicine, University of Montreal, Montreal, Quebec, Canada \\ ${ }^{4}$ Department of Genitourinary Medical Oncology, University of Texas, M.D. Anderson Cancer Center, Houston, Texas, USA \\ ${ }^{5}$ Cellular Biochemistry and Biophysics Program, Sloan-Kettering Institute for Cancer Research, New York, New York, USA \\ ${ }^{6}$ Division of Hematology and Medical Oncology, Department of Medicine, Weill Medical College of Cornell University, \\ New York, New York, USA \\ Address correspondence to: David M. Nanus, Weill Medical College of Cornell University, 525 E. 68th Street, \\ New York, New York 10021, USA. Phone: (212) 746-2920; Fax: (212) 746-6645; E-mail: dnanus@med.cornell.edu.
}

Received for publication June 8, 2000, and accepted in revised form October 31, 2000.

Neutral endopeptidase 24.11 (NEP, CD10) is a cell-surface enzyme expressed by prostatic epithelial cells that cleaves and inactivates neuropeptides implicated in the growth of androgen-independent prostate cancer (PC). NEP substrates such as bombesin and endothelin-1 induce cell migration. We investigated the mechanisms of NEP regulation of cell migration in PC cells, including regulation of phosphorylation on tyrosine of focal adhesion kinase (FAK). Western analyses and cell migration assays revealed an inverse correlation between NEP expression and the levels of FAK phosphorylation and cell migration in PC cell lines. Constitutively expressed NEP, recombinant NEP, and induced NEP expression using a tetracycline-repressive expression system inhibited bombesin- and endothelin-1-stimulated FAK phosphorylation and cell migration. This results from NEP-induced inhibition of neuropeptide-stimulated association of FAK with cSrc protein. Expression of a mutated catalytically inactive NEP protein also resulted in partial inhibition of FAK phosphorylation and cell migration. Coimmunoprecipitation experiments show that NEP associates with tyrosine-phosphorylated Lyn kinase, which then binds the p 85 subunit of phosphatidylinositol 3-kinase (PI3-K) resulting in an NEP-Lyn-PI3-K protein complex. This complex competitively blocks FAK-PI3-K interaction, suggesting that NEP protein inhibits cell migration via a protein-protein interaction independent of its catalytic function. These experiments demonstrate that NEP can inhibit FAK phosphorylation on tyrosine and PC cell migration through multiple pathways and suggest that cell migration which contributes to invasion and metastases in PC cells can be regulated by NEP.

J. Clin. Invest. 106:1399-1407 (2000).

\section{Introduction}

The molecular events involved in prostate cancer (PC) development and progression are not well defined. Recent data implicate neuropeptides such as bombesin, endothelin-1 (ET-1), and neurotensin in various stages of PC, including PC development, PC cell migration, and progression to hormone independence (1-3). Access of these neuropeptides to their cell-surface receptors is negatively regulated in part by neutral endopeptidase 24.11 (NEP, CD10), a 90-110 kDa zinc-dependent metallopeptidase that cleaves peptide bonds on the amino side of hydrophobic amino acids. The catalytic domain of NEP is located in the extracellular part of the NEP protein. NEP, which is expressed on benign prostate epithelial cells, normally functions to reduce local concentrations of neuropeptide available for receptor binding and signal transduction $(4,5)$. A decrease in NEP expression in PC cells may contribute to PC tumor progression by allowing neuropeptides to bind their receptors and provide growth stimulatory pathways (6).

NEP has been implicated in the inhibition of cell migration in neutrophils and lung fibroblasts $(7,8)$. In PC cells, NEP is highly expressed by LNCaP cells (6), a PC cell line that is often used as a model for hormonesensitive PC. LNCaP cells do not migrate or invade through ECM, in contrast to hormone-insensitive PC cell lines TSU-Pr1, DU145, or PC-3, which do not express NEP (6) and exhibit cell migration that can be increased by the NEP-substrate bombesin (2). This correlation in PC cells of loss of NEP expression and cell migration could be explained by the catalytic capability of NEP to inactivate neuropeptides such as bombesin which promote cell migration.

The biological and regulatory effects of NEP are presumed only to result from its enzymatic function (4, 5 ). However, recent data suggest that NEP may possess 
other biological properties in addition to its ability to catalytically inactivate neuropeptide substrates and that NEP protein expression in of itself can effect signal transduction pathways which regulate cell growth $(9,10)$ and apoptosis $(11)$. In the present study, we used LNCaP cells and hormone-independent TSU-Pr1 PC cells to investigate the mechanisms of NEP action by assessing the effects of NEP on regulating phosphorylation of focal adhesion kinase (FAK) on tyrosine and cell migration.

\section{Methods}

Cell culture and reagents. PC cell lines were maintained in RPMI 1640 media supplemented with $2 \mathrm{mM}$ glutamine, $1 \%$ nonessential amino acids, $100 \mathrm{U} / \mathrm{ml}$ streptomycin and penicillin, and $10 \%$ FCS. Reagents used include recombinant NEP (rNEP; Arris Pharmaceuticals Corp., South San Francisco, California, USA); CGS24592 (Novartis Pharmaceuticals, Summit, New Jersey, USA) (12); and PP2 (Calbiochem-Novabiochem Ltd., La Jolla, California, USA). The following antibodies were used: mouse monoclonals to NEP (J5; Beckman Coulter, Fullerton, California, USA); to Lyn (H-6), to cSrc (B-12), to anti-pTyr (PY20; Santa Cruz Biotechnology Inc., Santa Cruz, California, USA); rabbit polyclonals to NEP (5B5; Arris Pharmaceuticals Corp.), to p85 (Upstate Biotechnology, Lake Placid, New York, USA), to FAK (C-20), to Lyn (clone 44), and to cSrc (SRC-2; Santa Cruz Biotechnology Inc.).

Plasmid construction and gene transfer. TSU-GK27-NEP (WT-5) and TSU-GK27-Neo (TN-12) cells were constructed and maintained as described previously (6). A mutated NEP gene containing a substitution of Val for the catalytic Glu residue was constructed by PCR-based site-directed mutagenesis (13). A 1,525-bp PCR DNA fragment was cloned and sequenced entirely to confirm the presence of the mutation and the absence of other bp changes. A 1,339-bp DNA fragment containing the mutated region was used to replace the equivalent fragment in the expression vector PSVCALLA, which, when transfected into COS-1 cells, promoted the expression of high amounts of human NEP but no enzymatic activity, as reported previously for the same mutation in rabbit NEP (14). A DNA fragment containing the mutated sequence was used to replace the equivalent fragment in the pTRE-NEP vector. The pTRE-mutated NEP vector was then introduced into TSU-GK27 cells and stable cell lines expressing an inducible mutated NEP protein isolated (M-22 cells) as described elsewhere (6).

Enzyme assays. Cells were incubated with or without tetracycline, and NEP-specific enzyme activity assays were performed on total cell lysates as described previously (15) using Suc-Ala-Ala-Phe-pNA (Bachem Bioscience Inc., Philadelphia, Pennsylvania, USA) as substrate. Specific activities were expressed as picomoles per milligram of protein per minute and represent an average of two separate measurements performed in duplicate.

Immunoprecipitation and immunoblotting. Cells were lysed in $1 \mathrm{ml}$ of RIPA buffer (10 mM Tris- $\mathrm{HCl}$ [pH 7.4], $150 \mathrm{mM} \mathrm{NaCl}, 1 \%$ Triton X-100, 5 mM EDTA, $1 \%$ sodi- um deoxycholate, $0.1 \% \mathrm{SDS}, 1.2 \%$ aprotinin, $5 \mu \mathrm{M}$ leupeptin, $4 \mu \mathrm{M}$ antipain, $1 \mathrm{mM}$ phenylmethylsulfonyl fluoride, and $\left.0.1 \mathrm{mM} \mathrm{Na} 3 \mathrm{VO}_{4}\right) ; 300-500 \mu \mathrm{g}$ was incubated 1 hour to overnight with $1-4 \mu \mathrm{g}$ primary antibody and for 1 hour with $40 \mu \mathrm{l}$ protein G-Sepharose beads (Amersham Pharmacia Biotech, Piscataway, New Jersey, USA) at $4^{\circ} \mathrm{C}$; and immunoprecipitates were washed with RIPA buffer, resuspended in $2 x$ Laemmli sample buffer, resolved on $8 \%$ or $10 \%$ SDS-PAGE, and transferred to nitrocellulose. Western blot analyses were performed as described previously (15) using 1:2,000 dilution of antibody. For Far Western analysis, membranes were incubated with $1 \mu \mathrm{g} / \mathrm{ml} \mathrm{GST}$ fusion protein containing the $\mathrm{COOH}$-terminal Src-homology $(\mathrm{SH}) 2$ domain of p85 subunit of PI3-K (Upstate Biotechnology) for 2 hours and then Western blotted using the antiGST mAb (1:2,000; Santa Cruz Biotechnology Inc.) and anti-mouse secondary antibody. Relative intensities of each band were measured by NIH image and levels of phosphorylated protein relative to total protein calculated as the phosphorylation ratio. All experiments were performed on at least two separate occasions using different cell lysates with similar results.

Cell migration assays. Migration experiments were performed using $10 \mathrm{~mm}$ Tissue Culture Inserts with 8.0 $\mu \mathrm{m}$ polycarbonate membranes (Nalge Nunc, Naperville, Illinois, USA) as described elsewhere (2) with minor modifications. Membranes were coated with $100 \mu \mathrm{l}$ of $1 \mathrm{mg} / \mathrm{ml}$ of MATRIGEL matrix (Becton Dickinson Labware, Bedford, Massachusetts, USA) for 1 hour at room temperature, followed by $2 \%$ BSA in PBS as a blocking reagent for 1 hour. After overnight culture in serum-free media (LNCaP, TSU-Pr1, DU145, and PC-3) or culture for 48 hours in media containing tetracycline and/or CGS24592 (WT-5, $\mathrm{TN}-12$, and M-22), cells were harvested, washed, and resuspended in serum-free RPMI1640 and added to the upper chambers at concentrations of $5 \times 10^{4}$ cells per well. Media in upper and lower chambers contained serum-free RPMI1640 with bombesin or ET-1 at appropriate concentrations or $10 \% \mathrm{FCS}$ (LNCaP, TSU-Pr1, DU145, and PC-3); or serum-free RPMI1640 with or without tetracycline (WT-5, TN-12, and M-22). CGS24592, rNEP, and PP2 were added to cells in upper chambers and to the media in lower chambers 2 hours before other reagents. Cells were allowed to migrate for 10 hours at $37^{\circ} \mathrm{C}$ in a humidified atmosphere containing $5 \% \mathrm{CO}_{2}$. Membranes were fixed and stained using a Diff-Quik Stain Set (Dade Behring Inc., Deerfield, Illinois, USA). Nonmigrated cells on the upper side of the membrane were removed with a cotton swab. Migrated cells attached to the lower side of the membrane were enumerated using a light microscope at 10× magnification. Each data point represents the average cell number of six independent microscopic fields from a single experiment. Statistical analyses were performed using an unpaired $t$ test. All migration assays were performed on three separate occasions with similar results. 

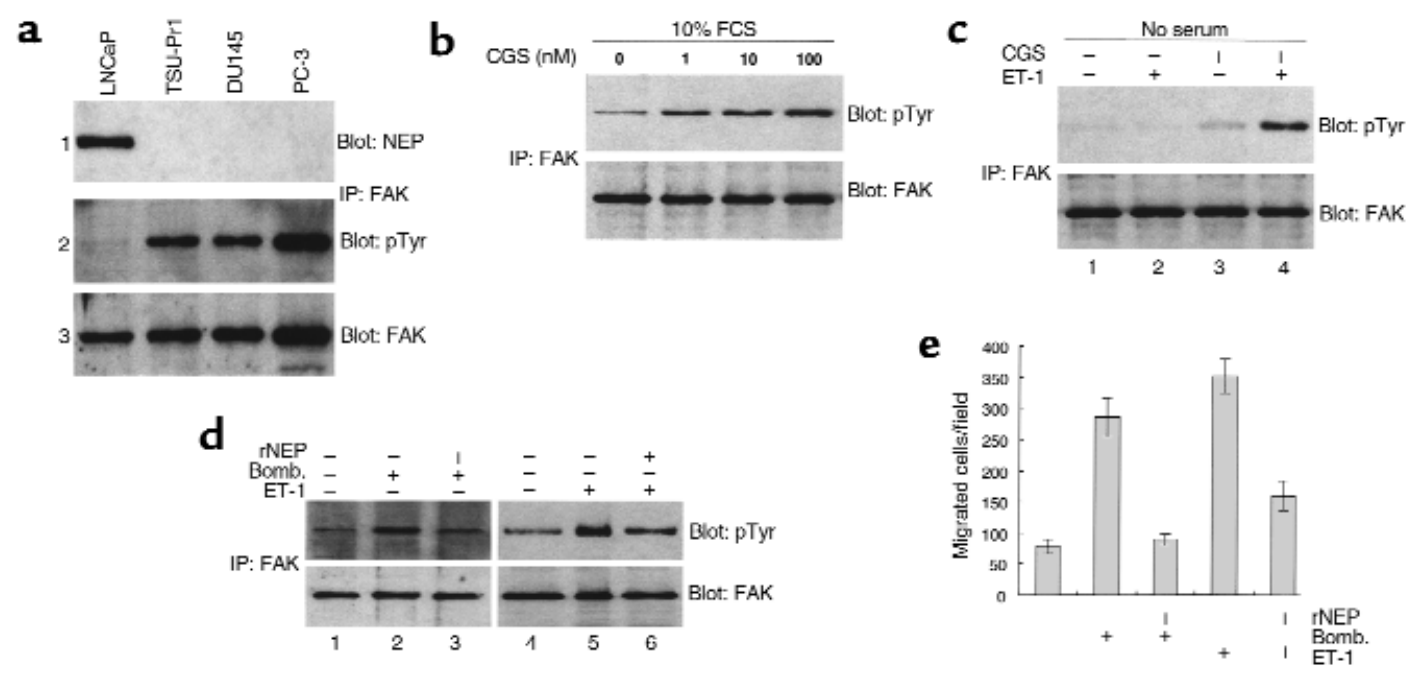

\begin{abstract}
Figure 1
NEP expression, FAK phosphorylation, and cell migration in PC cells. (a) Panel 1: total cell lysates $(20 \mu \mathrm{g})$ from PC cells were analyzed for NEP protein by Western blot as described in Methods using the anti-NEP antibody 5B5. Panel 2: $300 \mu \mathrm{g}$ of PC total cell lysates were immunoprecipitated (IP) with anti-FAK antibody C-20, separated by SDS-PAGE, transferred to nitrocellulose, and Western blotted with anti-pTyr mAb PY20. Panel 3: the same blot shown in panel 2 was stripped and reprobed with antibody C-20 for FAK protein. (b) LNCaP cells cultured in RPMI containing FCS and the NEP competitive enzyme inhibitor CGS24592 (CGS) for 2 hours at concentrations ranging from 0 to $100 \mathrm{nM}$ were immunoprecipitated with anti-FAK antibody C20, separated by SDS-PAGE, transferred to nitrocellulose, and Western blotted consecutively with mAb PY20 (upper panel) and antibody C-20 (lower panel). (c) LNCaP cells cultured in RPMI without serum (lane 1), $10 \mathrm{nM}$ ET-1 for 20 minutes (lane 2), 10 nM CGS24592 for 2 hours (lane 3), or 10 nM CGS24592 for 2 hours followed by 10 nM ET-1 for 20 minutes (lane 4). Cells were lysed and analyzed as described in b. (d) TSU-Pr1 cells were cultured in media without FCS for 24 hours (lanes 1 and 4 ), followed by the addition of $10 \mathrm{nM}$ bombesin (Bomb., lane 2) or $10 \mathrm{nM} \mathrm{ET-1}$ (lane 5) for 20 minutes; or by the addition of $50 \mu \mathrm{g} / \mathrm{ml}$ of rNEP for 2 hours, and then bombesin (lane 3) or 10 ET-1 (lane 6) for 20 minutes. Cells were lysed and analyzed as described in b. (e) Cell migration assays were performed in conditions identical to $\mathbf{d}$. Bars represent SD. Experiments were repeated three times with similar results.
\end{abstract}

\section{Results}

NEP enzymatic activity inhibits FAK phosphorylation and cell migration in PC cells. Focal adhesion kinase (FAK) is an important component of integrin-mediated signal transduction and plays a key role in modulating cell migration of PC cells $(2,16)$. FAK is activated through tyrosine phosphorylation, which occurs after cell adhesion to ECM proteins and ligand binding of growth factors to their cell-surface receptors, and FAK phosphorylation on tyrosine-397 is necessary for FAK-promoted cell migration (17). Anti-pTyr Western blotting of FAK immunoprecipitates revealed low levels of FAK phosphorylation and a slightly lower amount of total FAK protein in androgen-sensitive LNCaP cells compared with the levels of FAK phosphorylation and FAK protein in androgen-independent, TSU-Pr1, DU145, and PC-3 PC cells (Figure 1a, panels 2 and 3), which inversely correlated with the amount of NEP protein in these cells (Figure 1a, panel 1). TSU-Pr1, DU145, and PC-3 cells ability to migrate through Matrigel was 9.7, 9.1-, and 12.2-fold higher, respectively, then LNCaP cells $(P<0.005$ for all three cell lines).

NEP neuropeptide substrates bombesin and ET-1 stimulate phosphorylation of FAK $(18,19)$ and promote the migration of highly metastatic PC cells through ECM but have minimal effects on $\mathrm{LNCaP}$ cells (20). Inhibition of NEP enzymatic activity in LNCaP cells cultured in RPMI containing FCS using the NEP competitive enzyme inhibitor CGS24592 for 2 hours at concentrations ranging from 0 to $100 \mathrm{nM}$ resulted in increased FAK phosphorylation on tyrosine in a dose-dependent fashion (Figure 1b). Pretreatment of LNCaP cells cultured in RPMI without FCS with 10 nM CGS24592 for 2 hours before the addition of $10 \mathrm{nM} \mathrm{ET}-1$ for $20 \mathrm{~min}$ utes resulted in a 30-fold increase in FAK phosphorylation (Figure 1c, lane 4), compared with ET-1 or CGS24592 alone (Figure 1c, lanes 2 and 3). CGS24592 $(10 \mathrm{nM})$ incubation in RPMI containing FCS resulted in 4.3-fold increase in LNCaP migrated cell number compared with untreated control cells $(P<0.005)$.

Similar experiments investigating the effect of rNEP on FAK phosphorylation and cell migration in TSU-Pr1 cells cultured in media containing FCS showed rNEP can inhibit FAK phosphorylation and cell migration in a time and dose-dependent fashion (data not shown). Bombesin or ET-1 stimulate a 22and 26-fold increase, respectively, in the levels of phosphorylated FAK in TSU-Pr1 cells cultured in media without serum for 24 hours (Figure 1d, lanes 2 and 5), which is significantly inhibited by pretreatment with rNEP for 2 hours (Figure 1d, lanes 3 and 6). Furthermore, bombesin- and ET-1-induced cell migration of TSU-Pr1 cells (3.6-fold for bombesin, $P<0.005$; 4.4-fold for ET- $1, P<0.005)$ is blocked by rNEP (Figure 1e). Taken together, these data on LNCaP and TSU-Pr1 cells suggest that loss of NEP 
expression in PC cells results in increased FAK phosphorylation and cell migration, which is mediated by neuropeptide NEP substrates.

Overexpression of cell-surface NEP inhibits FAK phosphorylation and cell migration. To characterize further the role of cell-surface NEP in regulating FAK phosphorylation and cell migration, we used the inducible tetracycline-regulatory gene expression system to establish cell lines in parental TSU-Pr1 cells that express wild-type NEP (WT-5), catalytically inactive NEP (M-22), and a control cell line containing the pTRE empty vector (TN-12) (6). Western analysis showed that NEP protein was expressed in both WT- 5 and M-22 cells but not TN-12 cells 48 hours after tetracycline withdrawal (Figure 2a). Enzyme assays showed high levels of NEP-specific activity in total cell lysates from WT-5 cells $(505.3 \pm 82.7 \mathrm{pmol} / \mu \mathrm{g} / \mathrm{min})$ compared with minimal activity in lysates from TN-12 $(2.0 \pm 0.7 \mathrm{pmol} / \mu \mathrm{g} / \mathrm{min})$ or $\mathrm{M}-22 \quad(7.3 \pm 4.2$ $\mathrm{pmol} / \mathrm{\mu g} / \mathrm{min}$ ) cells after tetracycline withdrawal. NEP expression in WT- 5 cells resulted in an approximately 95\% decrease in FAK phosphorylation (Figure 2b, lane 3 compared with lane 1), but did not alter FAK phosphorylation in TN-12 cells (Figure $2 \mathrm{~b}$, lane 6 compared with lane 5). However, in M-22 cells, induced expression of catalytically inactive NEP also resulted in a partial inhibition of FAK phosphorylation (Figure 2b, lane 8 compared with lane 7). Likewise, incubation of WT-5 cells after tetracycline withdrawal with 100 nM CGS24592 only partially inhibited FAK phosphorylation (Figure $2 b$, lane 4) despite that NEP enzymatic activity is completely inhibited by CGS24592 at this concentration (NEP enzyme-specific activity $6.5 \pm 3.3 \mathrm{pmol} / \mathrm{\mu g} / \mathrm{min}$ ). CGS24592 did not alter FAK phosphorylation when NEP expression was suppressed by tetracycline (Figure $2 \mathrm{~b}$, lane 2). As illustrated in Figure 2c, cell migration studies performed using the identical conditions depicted in Figure $2 \mathrm{~b}$ demonstrated that expression of NEP in WT- 5 cells resulted in a greater than $90 \%$ decrease compared with control $(P<0.005)$, which was partially reversed by $100 \mathrm{nM}$ CGS24592. NEP expression in M-22 cells resulted in a $54 \%$ decrease $(P<0.005)$, which was less than that observed in WT- 5 cells but comparable to the inhibition observed in WT-5 cells cultured with CGS24592. These data show that blocking NEP enzyme activity through either a competitive enzyme inhibitor or gene mutation does not completely abrogate the ability of cell-surface NEP to inhibit FAK phosphorylation and cell migration and suggest that expression of the NEP protein at the cell surface can induce dephosphorylation of FAK through a mechanism distinct from NEP's catalytic function.

NEP enzyme activity inbibits the formation of $c \mathrm{Src}$ and FAK protein complexes. The ability of FAK to transmit signals to downstream targets is dependent on its interaction with several intracellular signaling molecules including Src family kinases $(21,22)$ and PI3-K (23). Tyr-397 has been identified as the major site of FAK autophosphorylation and the binding site for the SH2 domains of $\mathrm{cSrc}$ (24) and the p85 subunit of PI3-
K (23). Substitution of Tyr-397 with Phe abolishes FAK-promoted cell migration (17), implicating a role for cSrc and PI3-K in cell migration. The association of FAK with cSrc is also critical in promoting bombesin-mediated FAK phosphorylation and cell migration via $G$ protein-coupled receptor (GPCR) pathways $(19,25)$. Western blotting of cSrc immunoprecipitates from PC cells revealed that FAK coimmunoprecipitates with cSrc in three androgen-independent PC cell lines but not in LNCaP cells (Figure 3a), despite the fact that LNCaP cells express abundant amounts of cSrc protein (Figure 3a, lower panel) and FAK protein (Figure 1a, panel 3). The FAK-cSrc coimmunoprecipitation was partially restored by culturing LNCaP cells in media containing FCS with CGS24592 (data not shown), or in serum-free media with CGS24592 followed by ET-1 (Figure 3b, lane 4). In TSU-Pr1 cells, the specific Src kinase inhibitor PP2 inhibited bombesin-induced FAK phosphorylation (Figure 3c, lane 3 compared with lane 2). A total of 10

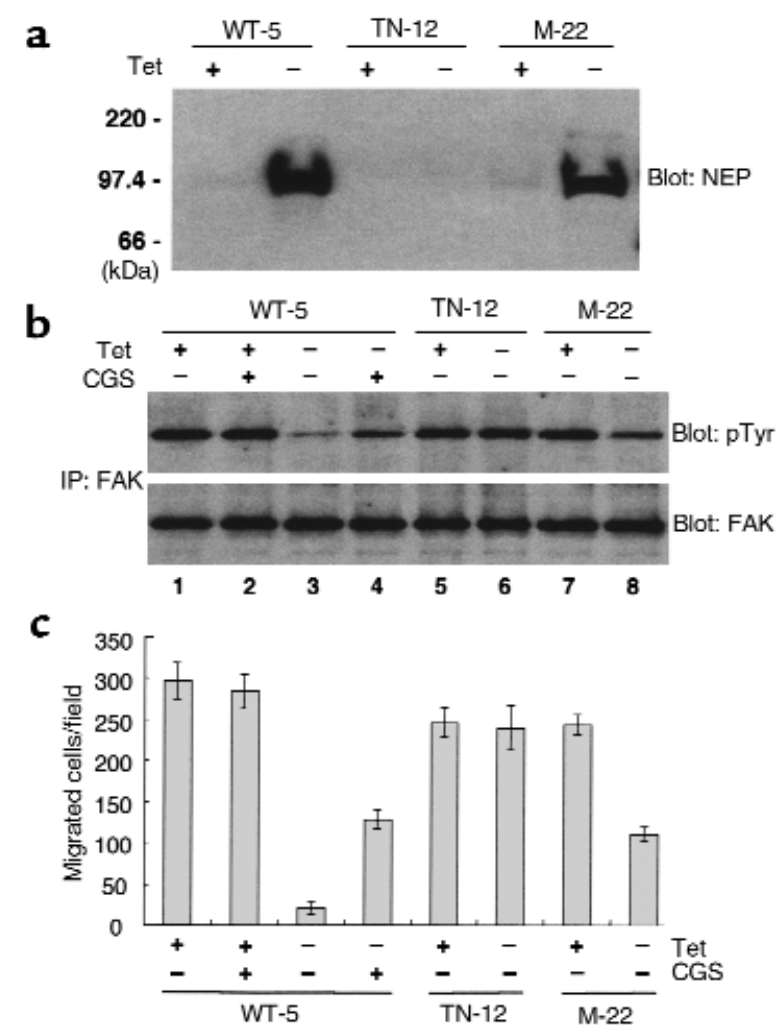

Figure 2

Overexpression of cell-surface NEP inhibits FAK phosphorylation and cell migration. (a) TSU-Pr1-derived cell lines containing wild-type NEP (WT-5), control empty vector (TN-12), or mutated, enzymatically inactive NEP (M-22) were cultured with $(+)$ and without $(-) 1$ $\mu \mathrm{g}$ tetracycline $(\mathrm{Tet})$. Total cell lysates $(20 \mu \mathrm{g})$ were analyzed for NEP protein by Western blot as described in Methods using the anti-NEP antibody 5B5. (b) WT-5, TN-12, and M-22 cells were cultured with $\left(^{+}\right)$and without $(-) 1 \mu \mathrm{g}$ tetracycline (with the addition of $100 \mathrm{nM}$ CGS24592 in lanes 2 and 4). Cells were lysed and analyzed as described in Figure 1b. (c) Cell migration assays were performed using the identical conditions described in $\mathbf{b}$. Bars represent SD. Experiments were repeated twice with similar results. 
$\mu \mathrm{M}$ PP2 also inhibited FAK phosphorylation in TSU-Pr1 cells cultured in media containing FCS (Figure $3 c$, lane 5 compared with lane 4). This decrease in FAK phosphorylation was not affected by the addition of $50 \mu \mathrm{g} / \mathrm{ml}$ of rNEP for 2 hours before the addition of PP2 (Figure 3c, lane 6). Cell migration assays showed that treatment of TSU-Pr1 cells with $10 \mu \mathrm{M}$ PP2 resulted in $45 \%$ decrease in migrated cell number compared with untreated control $(P=0.0025)$, and that the addition of $50 \mu \mathrm{g} / \mathrm{ml} \mathrm{rNEP}$ did not significant affect the migrated cell number compared with PP2 treatment $(P=0.3907)$. Finally, assessment of the association of cSrc and FAK in WT-5, M-22, and TN12 cells before and after tetracycline withdrawal showed that FAK and cSrc coimmunoprecipitated in WT-5 (Figure $3 \mathrm{~d}$, lane 1), M-22 (Figure 3d, lane 7) and TN-12 cells (Figure 3d, lane 5) when cultured in tetracycline, which suppresses NEP expression. Expression of NEP by tetracycline withdrawal completely inhibited the association of FAK and cSrc in WT-5 cells (Figure $3 \mathrm{~d}$, lane 3), but not in M22 cells (Figure 3d, lane 8). The inhibition of FAK-cSrc coimmunoprecipitation in WT-5 cells (Figure 3d, lane 3) was reversed by culturing in the presence of CGS24592 (Figure 3d, lane 4). Taken together, these data show that (a) NEP inhibition of neuropeptide-mediated FAK phosphorylation and cell migration results in part from lack of association of FAK with c-Src; (b) neuropeptide induction of FAK phosphorylation requires cSrc-kinase activity; (c) catalytic inactivation of NEP substrates does not further promote FAK dephosphorylation or inhibition of cell migration if cSrc-kinase activity is inhibited; and (d) the association of FAK and cSrc is inhibited by expression of enzymatically active NEP protein, but not by expression of catalytically inactive NEP protein.

NEP complexes with the $p 85$ subunit of PI3-K. Expression of catalytically inactive NEP protein in M22 cells partially inhibited FAK phosphorylation and cell migration, but did not alter the association of FAK and cSrc, suggesting an alternative pathway of NEP regulation of FAK phosphorylation and cell migration. Previous reports indicate that PI3-K interacts with and phosphorylates FAK in FAK-promoted cell migration (26). Western analysis of immunoprecipitates of the p85 subunit of PI3-K showed that p85 coimmunoprecipitated with FAK in androgen-independent PC cells but not in LNCaP cells (Figure 4a). In contrast to the association of FAK with $\mathrm{CSrc}$, the FAK-p85 coimmunoprecipitation could not be restored by incubating LNCaP cells with CGS24592 or CGS24592 and ET-1, indicating that this proteinprotein interaction was not dependent on NEP enzyme activity or NEP substrates (data not shown). Expression of wild-type NEP (Figure 4b, lane 3) or mutated NEP (Figure 4b, lane 8) blocked the association of $\mathrm{p} 85$ with FAK, which could not be reversed by culturing in the presence of the NEP enzyme inhibitor CGS24592 (Figure 4b, lane 4). These experiments showed NEP expression inhibits the associa- a

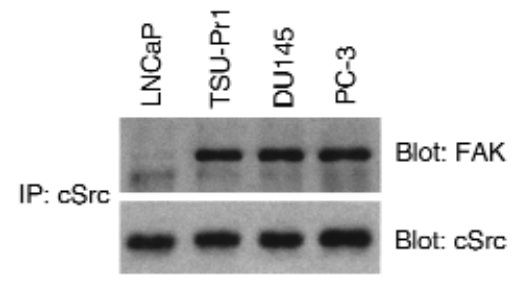

b

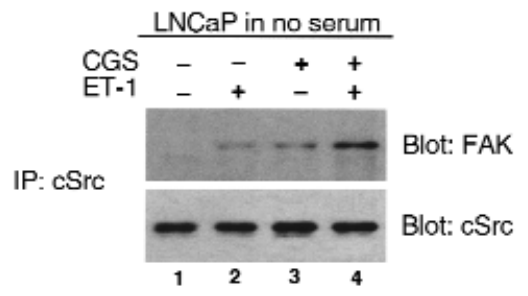

c
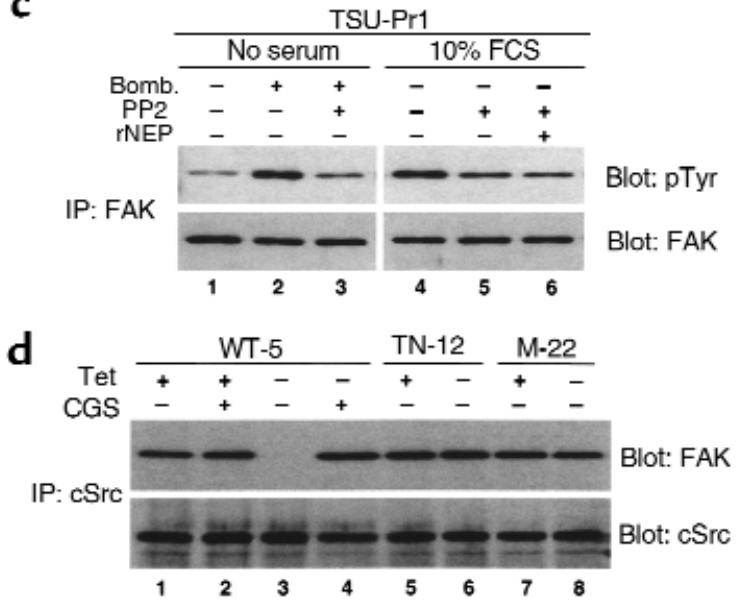

Figure 3

NEP enzyme activity inhibits the formation of cSrc and FAK protein complexes. (a) Upper panel: $500 \mu \mathrm{g}$ of PC total cell lysates were immunoprecipitated with anti-cSrc antibody SRC-2, separated by SDS-PAGE, transferred to nitrocellulose and Western blotted with anti-FAK antibody C20. (a) Lower panel: The same blot was stripped and reprobed with mAb B12 for cSrc protein. (b) Cell lysates derived from LNCaP cells cultured in media without FCS (lane 1), $10 \mathrm{nM}$ ET-1 for 20 minutes (lane 2), 10 nM CGS24592 for 2 hours (lane 3) or CGS24592 followed by ET-1 (lane 4) were analyzed as described in a. (c) (left) TSU-Pr1 cells were cultured in serum-free media (lane 1) with the addition of $10 \mathrm{nM}$ bombesin for 20 minutes (lane 2), or $10 \mu \mathrm{M}$ of Src kinase inhibitor PP2 for 30 minutes prior to the addition bombesin (lane 3). (right) TSU-Pr1 cells were cultured in media containing $10 \%$ FCS (lane 4 ), $10 \mu \mathrm{M}$ of PP2 (lane 5 ), or $50 \mu \mathrm{g} / \mathrm{ml}$ of rNEP for 2 hours prior to PP2. Cells were lysed and analyzed as described in Figure 1b. (d) WT-5, TN-12, and M-22 cells were cultured with (+) and without (-) $1 \mu \mathrm{g}$ tetracycline for 48 hours (with the addition of $100 \mathrm{nM}$ CGS24592 in lanes 2 and 4). Cells were lysed and analyzed as described in $\mathbf{a}$.

tion of p85 with FAK. Western blotting using an NEP antibody of immunoprecipitates of the p85 subunit of PI3-K revealed NEP coimmunoprecipitated with p85 in LNCaP cells but not other PC cell lines (Figure $5 a$, panel 1), which was confirmed by Western blotting of NEP immunoprecipitates using a p85 antibody (Figure 5a, panel 4). Identical results were obtained in WT-5 and M-22 cells in which NEP and 
a
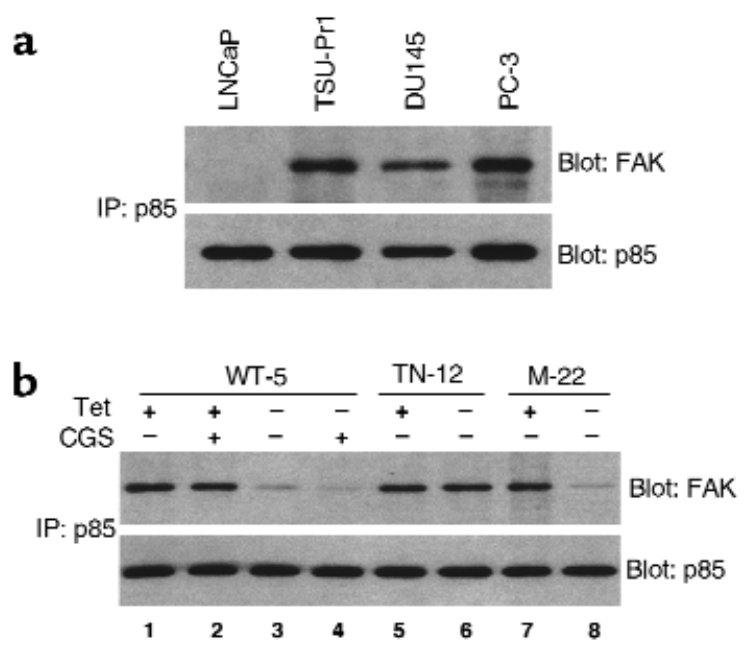

Figure 4

NEP inhibits the formation of p85-FAK protein complexes. (a) $500 \mu \mathrm{g}$ of total cell lysates were immunoprecipitated with anti-p85 Ab, separated by SDS-PAGE, transferred to nitrocellulose, and Western blotted with anti-FAK antibody C-20 or anti-p85 antibody. (b) WT-5, $\mathrm{TN}-12$, and $\mathrm{M}-22$ cells were cultured with (+) and without (-) $1 \mu \mathrm{g}$ tetracycline (with the addition of 100 nM CGS24592 in lanes 2 and 4). Cells were lysed and analyzed as described in a.

p85 coimmunoprecipitates could be detected when either wild-type or mutated NEP was expressed after tetracycline withdrawal (Figure $5 \mathrm{~b}$, panels 1 and 4 ). Thus, these data suggest that NEP protein complexes with the p85 subunit of PI3-K and inhibits the association of $\mathrm{p} 85$ with FAK, and that expression of catalytically inactive NEP protein in M22 cells partially inhibits FAK phosphorylation and cell migration by NEP complexing with p85.

Lyn kinase mediates p85-NEP interaction. NEP's cytoplasmic domain lacks any characteristic structural motifs such as an $\mathrm{SH} 2$ domain or a proline-rich region required for associating with p85, suggesting that NEP and p85 do not directly associate. NEP is reported to associate with the Src family kinase member Lyn and an unknown $75-$ to $85-\mathrm{kDa}$ protein in B cells $(9,10)$. Our study showed that Lyn was also detected by Western blotting of NEP immunoprecipitates with a Lyn antibody in NEP-expressing LNCaP, WT-5 and M-22 cells (data not shown). Similarly, Western blotting of Lyn immunoprecipitates with an NEP antibody detected NEP (Figure 6, $a$ and $b$, panel 1). Moreover, p85 coimmunoprecipitated with NEP and Lyn (Figure 6, a and b, panel 2), and antipTyr Western blotting of Lyn immunoprecipitates showed that Lyn phosphorylation is increased in cells expressing NEP (Figure 6, a and b, panel 3 compared with total Lyn protein in panel 4). Lyn directly associates with $\mathrm{p} 85$ when NEP is expressed as shown by binding of GST-p85 SH2 to Lyn immunoprecipitated from M-22 cells expressing NEP after tetracycline withdrawal (Figure 6c). Finally, an analysis of immunoprecipitates of p85 from M-22 cells at 8, 16, and 24 hours after tetracycline withdrawal indicated that the p85-FAK association
(Figure 6d, panel 1) decreased as the association of p85 with both NEP (Figure 6d, panel 2) and Lyn (Figure 6d, panel 3) increased. Taken together, these results suggest that NEP associates with and induces Lyn phosphorylation, which in turn directly binds to $\mathrm{p} 85$ of PI3-K, resulting in complex of NEP-Lyn-PI3-K, which competitively blocks FAK-PI3-K interaction, leading to a decrease in FAK phosphorylation and cell migration.

\section{Discussion}

The involvement of NEP loss in the transition from androgen-dependent PC to androgen-independent PC has only recently been recognized (6). We previously reported that NEP expression is decreased in many androgen-independent PCs in vivo (6); that the NEP gene is transcriptionally activated by androgen and that androgen withdrawal results in decreased NEP expression $(6,15)$; and that hypermethylation of the $5^{\prime}$ CPG NEP island in the NEP promoter is asso-
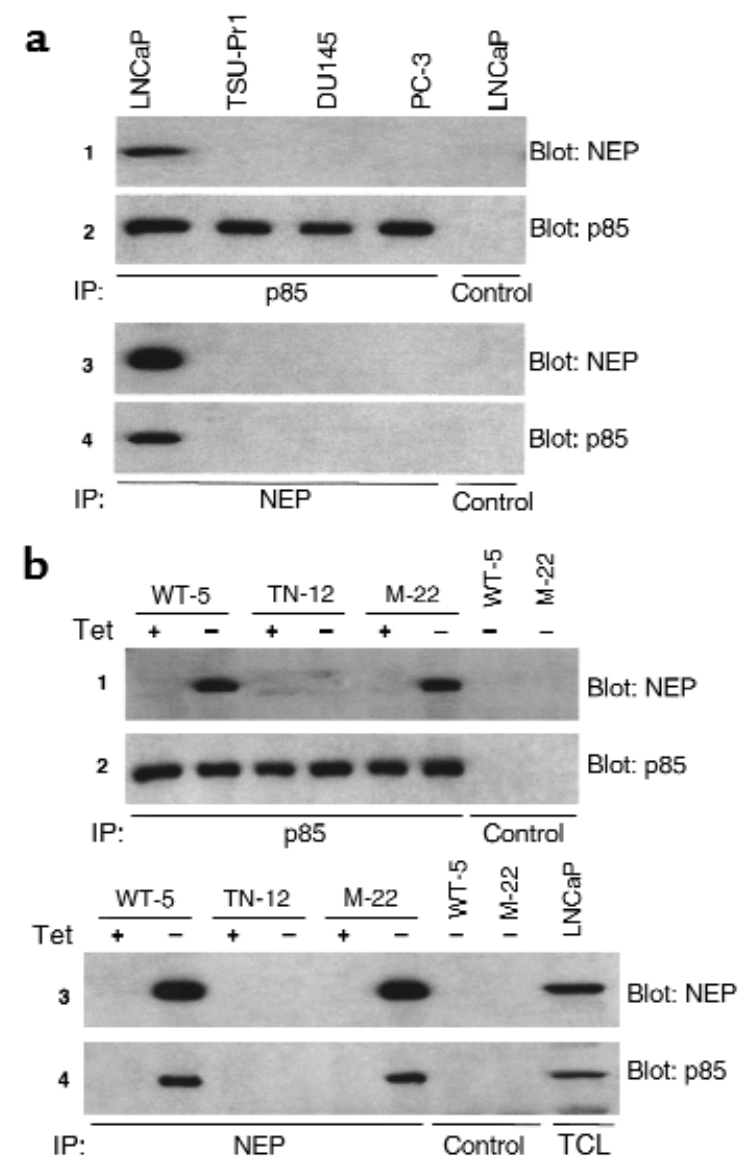

\section{Figure 5}

NEP complexes with the p85 subunit of PI3-K. (a) $500 \mu \mathrm{g}$ of total cell lysates were immunoprecipitated with anti-p85 antibody, anti-NEP antibody 5 or control antibody, separated by SDS-PAGE, transferred to nitrocellulose, and Western blotted with anti-NEP antibody 5B5 or anti-p85 antibody. (b) Cell lysates derived from WT-5, TN-12, and M-22 cells cultured with (+) and without $(-) 1 \mu \mathrm{g}$ tetracycline were immunoprecipitated with anti-p85 antibody, anti-NEP antibody 5 or control antibody and analyzed as described in $\mathbf{a}$. Total cell lysate (TCL) from LNCaP cells was positive control. 

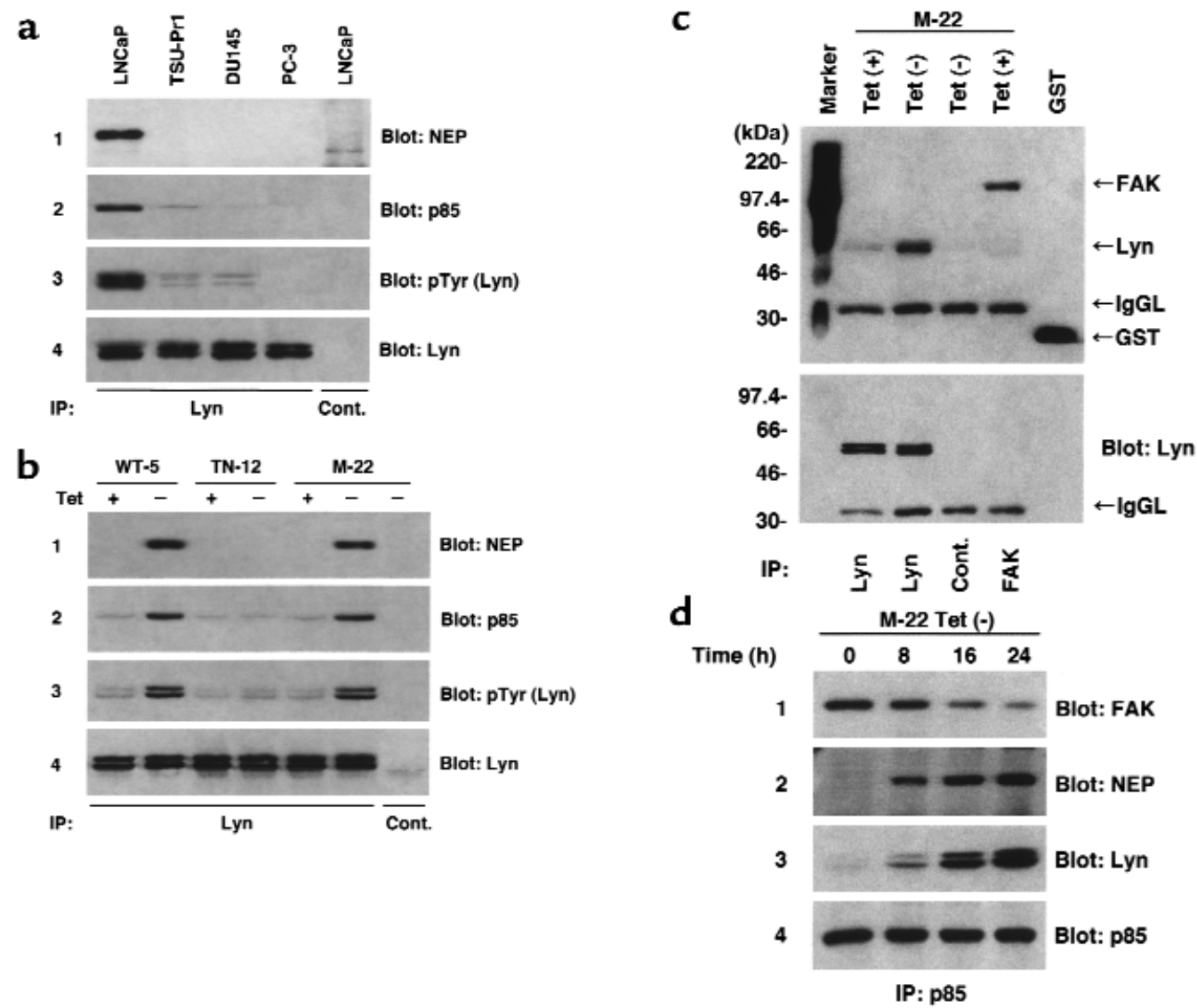

\begin{abstract}
Figure 6
Phosphorylated Lyn associates with NEP and p85. (a) $500 \mu \mathrm{g}$ of total cell lysates were immunoprecipitated with rabbit anti-Lyn antibody or control rabbit antibody, separated by SDS-PAGE, transferred to nitrocellulose, and Western blotted with anti-NEP antibody, anti-p85 antibody, anti-pTyr antibody or mouse anti-Lyn antibody. (b) WT-5, TN-12, and M-22 cells were cultured with (+) and without (-) $1 \mu \mathrm{g}$ tetracycline. Cells were lysed and analyzed as described in a. (c) Upper panel: M-22 cells were cultured with or without (-) $1 \mu \mathrm{g}$ tetracycline for 48 hours. Cells were lysed and Far Western blotted as described in Methods. FAK immunoprecipitate of M-22 cells was used as a positive control for $\mathrm{COOH}$-terminal SH2 domain of $\mathrm{p} 85$ to bind. GST; $0.1 \mu \mathrm{g}$ recombinant GST protein used as a positive control for anti-GST antibody. Cont.; negative control by rabbit control antibody. IgGL; IgG light chain. (c) Lower panel: the same blot shown in upper panel was stripped and reprobed with mouse anti-Lyn antibody. (d) M-22 cells were cultured without (-) $1 \mu \mathrm{g}$ tetracycline for various time periods. Cell lysates were immunoprecipitated with rabbit anti-p85 antibody, separated by SDS-PAGE, transferred to nitrocellulose, and Western blotted with anti-FAK antibody, anti-NEP antibody, mouse anti-Lyn antibody, or anti-p85 antibody.
\end{abstract}

ciated with loss of NEP expression (27). These results suggest a model in which decreased NEP expression is facilitated by the elimination of androgens or hypermethylation of the NEP promoter, contributing to the development of neuropeptide-mediated, androgen-independent PC cell growth. The current study aimed at elucidating the mechanisms of NEP action on PC cells reveals a dual function of the NEP protein (Figure 7). NEP inhibits neuropeptide-mediated stimulation of the association of cSrc and FAK through catalytic inhibition of its neuropeptide substrates such as bombesin and ET-1, and NEP inhibits the association of PI3-K with FAK by indirectly associating with PI3-K through Lyn kinase. These effects on FAK phosphorylation directly correlate with PC cell migration, and suggest that NEP normally functions to regulate PC cell migration and possibly other FAKassociated processes.
The effects of NEP on bombesin and ET- 1 are not surprising as these neuropeptides induce FAK activation via a cSrc-dependent pathway $(19,28)$. The association of FAK with cSrc plays a major role in FAK-mediated signal transduction (29), inducing phosphorylation of FAK at multiple sites, including Tyr 577 and Tyr 925, resulting in maximal FAK activity $(29,30)$. We show that cell migration, FAK phosphorylation and the association of cSrc with FAK (a) increases in NEP-negative, androgen-independent PC cells treated with neuropeptide; (b) increases in NEP-expressing LNCaP cells cultured with a specific NEP enzyme inhibitor plus neuropeptide; and (c) decreases with the addition of rNEP or the expression of cell-surface NEP in androgen-independent PC cells. These results suggest that NEP enzymatic activity inhibits FAK phosphorylation and cell migration by affecting neuropeptide-induced interaction of FAK with cSrc. 


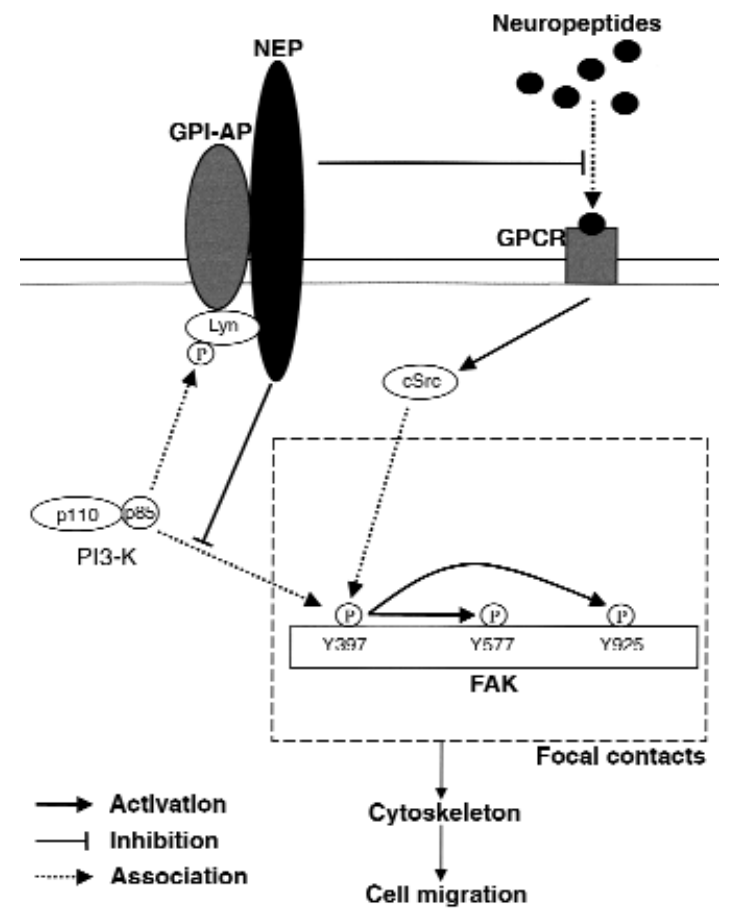

Figure 7

NEP effect on FAK-mediated cell migration. Neuropeptides such as bombesin and ET-1 promote the association of cSrc with FAK, resulting in phosphorylation of FAK at multiple sites including Tyr 577 and Tyr 925. NEP catalytically inactivates these neuropeptides and inhibits neuropeptide-induced, FAK-mediated cell migration. PI3-K also associates with FAK in focal contacts which may further activate FAK-mediated cell migration. NEP complexes with phosphorylated Lyn and binds the $\mathrm{p} 85$ subunit of PI3-K, competitively inhibiting the association of $\mathrm{p} 85$ with FAK, resulting in decreased FAK-mediated cell migration. GPI-AP, GPI-anchored protein.

Expression of a mutated, catalytically inactive cellsurface NEP protein also inhibited FAK phosphorylation and cell migration although to a lesser degree than observed following expression of wild-type NEP. This observation suggested another mechanism of NEP action distinct from its enzymatic function, and led us to examine whether PI3-K, which has been implicated in promoting cell migration, was involved. The concept that NEP protein independent of its catalytic activity is involved in signal transduction is supported by two recent studies. NEP/CD10 expression is a marker for apoptosis in $\mathrm{T}$ cells and the apoptotic capacity is not dependent of NEP catalytic activity (11), and NEP/CD10 expression negatively affects integrin-mediated signal transduction in NEP/CD10 positive B lymphocyte leukemia cells (31). Our observation that NEP protein associates with the $\mathrm{p} 85$ subunit of PI3-K was unexpected. This association occurs in LNCaP cells and in androgen-independent PC cells in which NEP expression is induced. Expression of NEP in these cells is also associated with a loss of the interaction of $\mathrm{p} 85$ with FAK. Numerous studies have suggested that FAK associates with PI3-K $(23,26,32)$ and this association affects the integrity of the actin cytoskeleton (32) and cell migration (26), although it remains controversial whether PI3-K-mediated cell migration is dependent on $\operatorname{FAK}(26,33)$. Thus, we hypothesize that in PC cells NEP's effect on cell migration and FAK phosphorylation is also mediated by inhibition of FAK-PI3-K interaction.

Although we have shown that tyrosine-phosphorylated Lyn that is induced following NEP expression inhibits FAK-PI3-K interactions by associating directly with $\mathrm{p} 85$, we did not define the enzyme that phosphorylates Lyn, or how an NEP-phosphorylated Lyn complex inhibits PI3-K associating with FAK. NEP is present in detergent-resistant glycosylphosphatidylinositol (GPI) microdomains (10), which are enriched in various signaling molecules, including p 85 and its substrate PIP2 (34). NEP in GPI microdomains coimmunoprecipitates with Lyn and an unknown 75- to $85-\mathrm{kDa}$ protein $(9,10)$, which we speculate is $\mathrm{p} 85$. It is unclear whether the intracellular domain of NEP is necessary for these interactions; however, GPI-anchored cell-surface proteins lacking an intracellular domain coprecipitate with other Src-related protein tyrosine kinases (35), suggesting that the intracellular domain of NEP may not be required. PI3-K located in focal contacts can reactivate integrin function through inside-out signaling $(36,37)$, suggesting that PI3-K associates with FAK in focal contacts and also regulates FAK-mediated cell migration pathways. In addition, Lyn has previously been implicated as a negative regulator of PI3-K signaling $(38,39)$. Thus, we speculate that NEP expression results in phosphorylated Lyn binding PI3-K causing PI3-K to translocate from focal contacts (which contain integrin-FAK complexes), thereby inhibiting FAK-PI3-K interactions. Preliminary studies support this hypothesis (our unpublished data).

In summary, we describe a novel dual function of NEP to inhibit FAK phosphorylation and cell migration by blocking FAK association with cSrc and PI3-K, suggesting a complex system in which NEP plays an important role. Recent studies showing that bombesin signaling cross-talks with the EGF receptor (40), and that ET-1 acts as a survival factor (41), suggest that NEP may be involved in other critical pathways. Further studies will help define the role of NEP and its substrates in regulating PC development and progression.

\section{Acknowledgments}

We thank Satoshi Suzuki and Badar Usmani for useful discussions, Michele Letarte at the Hospital for Sick Children in Toronto for supplying human NEP cDNA, and Catherine Kearney and Lana Winter for secretarial assistance. This work was supported by NIH grant CA-80240, the Association for the Cure of Cancer of the Prostate (CaP CURE), and the Dorothy Rodbell Foundation for Sarcoma Research. J. Dai is a recipient of a Department of Defense Prostate Cancer Research Program Postdoctoral Traineeship Award. 
1. Sehgal, I., et al. 1994. Neurotensin is an autocrine trophic factor stimulated by androgen withdrawal in human prostate cancer. Proc. Natl. Acad. Sci. USA. 91:4673-4677.

2. Aprikian, A.G., Tremblay, L., Han, K., and Chevalier, S. 1997. Bombesin stimulates the motility of human prostate-carcinoma cells through tyrosine phosphorylation of focal adhesion kinase and of integrin-associated proteins. Int. J. Cancer. 72:498-504.

3. Nelson, J.B., and Carducci, M.A. 2000. Small bioactive peptides and cell surface peptidases in androgen-independent prostate cancer. Cancer Invest. 18:87-96.

4. Shipp, M.A., et al. 1991. CD10/neutral endopeptidase 24.11 hydrolyzes bombesin-like peptides and regulates the growth of small cell carcinomas of the lung. Proc. Natl. Acad. Sci. USA. 88:10662-10666.

5. Shipp, M.A., and Look, A.T. 1993. Hematopoietic differentiation antigens that are membrane-associated enzymes: cutting is the key! Blood. 82:1052-1070.

6. Papandreou, C.N., et al. 1998. Neutral endopeptidase 24.11 loss in metastatic human prostate cancer contributes to androgen-independent progression. Nat. Med. 4:50-57.

7. Harrison, N.K., et al. 1995. Effects of neuropeptides on human lung fibroblast proliferation and chemotaxis. Am. J. Physiol. 268:L278-L283.

8. Hofman, P., et al. 1998. CD10 inhibitors increase f-Met-Leu-Phe-induced neutrophil transmigration. J. Leukoc. Biol. 63:312-320.

9. Ganju, R.K., Shpektor, R.G., Brenner, D.G., and Shipp, M.A. 1996. CD10/neutral endopeptidase 24.11 is phosphorylated by casein kinase II and coassociates with other phosphoproteins including the lyn srcrelated kinase. Blood. 88:4159-4165.

10. Angelisova, P., Drbal, K., Horejsi, V., and Cerny, J. 1999. Association of CD10/neutral endopeptidase 24.11 with membrane microdomains rich in glycosylphosphatidylinositol-anchored proteins and Lyn kinase. Blood. 93:1437-1439.

11. Cutrona, G., et al. 1999. Expression of CD10 by human T cells that undergo apoptosis both in vitro and in vivo. Blood. 94:3067-3076.

12. De Lombaert, S., et al. 1994. N-Phosphonomethyl dipeptides and their phosphonate prodrugs, a new generation of neutral endopeptidase (NEP, EC 3.4.24.11) inhibitors. J. Med. Chem. 37:498-511.

13. Yang, X.F., Chatellard, C., Lazure, C., Crine, P., and Boileau, G. 1994 Insertion of hydrophilic amino acid residues in the signal peptide/membrane anchor domain of neprilysin (neutral endopeptidase 24.11) results in its cleavage: role of the position of insertion. Arch. Biochem. Biophys. 315:382-386

14. Devault, A., et al. 1988. Expression of neutral endopeptidase (enkephalinase) in heterologous COS-1 cells. Characterization of the recombinant enzyme and evidence for a glutamic acid residue at the active site. J. Biol. Chem. 263:4033-4040.

15. Shen, R., et al. 2000. Androgen-induced growth inhibition of androgen receptor expressing androgen-independent prostate cancer cells is mediated by increased levels of neutral endopeptidase. Endocrinology. 141:1699-1704

16. Zheng, D.Q., Woodard, A.S., Fornaro, M., Tallini, G., and Languino, L.R. 1999. Prostatic carcinoma cell migration via alpha(v)beta3 integrin is modulated by a focal adhesion kinase pathway. Cancer Res. 59:1655-1664.

17. Cary, L.A., Chang, J.F., and Guan, J.L. 1996. Stimulation of cell migration by overexpression of focal adhesion kinase and its association with Src and Fyn. J. Cell Sci. 109:1787-1794.

18. Zachary, I., and Rozengurt, E. 1992. Focal adhesion kinase (p125FAK): a point of convergence in the action of neuropeptides, integrins, and oncogenes. Cell. 71:891-894.

19. Salazar, E.P., and Rozengurt, E. 1999. Bombesin and platelet-derived growth factor induce association of endogenous focal adhesion kinase with Src in intact Swiss 3T3 cells. J. Biol. Chem. 274:28371-28378.

20. Tremblay, L., et al. 1996. Focal adhesion kinase (pp125FAK) expression, activation and association with paxillin and p50CSK in human metastatic prostate carcinoma. Int. J. Cancer. 68:164-171.
21. Schlaepfer, D.D., Hanks, S.K., Hunter, T., and van der Geer, P. 1994. Integrin-mediated signal transduction linked to Ras pathway by GRB2 binding to focal adhesion kinase. Nature. 372:786-791.

22. Schaller, M.D., Hildebrand, J.D., and Parsons, J.T. 1999. Complex formation with focal adhesion kinase: a mechanism to regulate activity and subcellular localization of Src kinases. Mol. Biol. Cell. 10:3489-3505.

23. Chen, H.C., Appeddu, P.A., Isoda, H., and Guan, J.L. 1996. Phosphorylation of tyrosine 397 in focal adhesion kinase is required for binding phosphatidylinositol 3-kinase. J. Biol. Chem. 271:26329-26334.

24. Schaller, M.D., et al. 1994. Autophosphorylation of the focal adhesion kinase, pp125FAK, directs SH2-dependent binding of pp60src. Mol. Cell. Biol. 14:1680-1688.

25. Rozengurt, E. 1998. Signal transduction pathways in the mitogenic response to G protein-coupled neuropeptide receptor agonists. J. Cell. Physiol. 177:507-517.

26. Reiske, H.R., et al. 1999. Requirement of phosphatidylinositol 3-kinase in focal adhesion kinase-promoted cell migration. J. Biol. Chem. 274:12361-12366.

27. Usmani, B.A., et al. 2000. Methylation of the neutral endopeptidase gene promoter in human prostate cancers. Clin. Cancer Res. 6:1664-1670.

28. Rodriguez-Fernandez, J.L., and Rozengurt, E. 1998. Bombesin, vasopressin, lysophosphatidic acid, and sphingosylphosphorylcholine induce focal adhesion kinase activation in intact Swiss 3T3 cells. J. Biol. Chem. 273:19321-19328.

29. Schlaepfer, D.D., Jones, K.C., and Hunter, T. 1998. Multiple Grb2-mediated integrin-stimulated signaling pathways to ERK2/mitogen-activated protein kinase: summation of both c-Src- and focal adhesion kinaseinitiated tyrosine phosphorylation events. Mol. Cell. Biol. 18:2571-2585.

30. Calalb, M.B., Polte, T.R., and Hanks, S.K. 1995. Tyrosine phosphorylation of focal adhesion kinase at sites in the catalytic domain regulates kinase activity: a role for Src family kinases. Mol. Cell. Biol. 15:954-963.

31. Geijtenbeek, T.B., et al. 1999. High frequency of adhesion defects in Blineage acute lymphoblastic leukemia. Blood. 94:754-764.

32. Guinebault, C., et al. 1995. Integrin-dependent translocation of phosphoinositide 3-kinase to the cytoskeleton of thrombin-activated platelets involves specific interactions of $\mathrm{p} 85$ alpha with actin filaments and focal adhesion kinase. J. Cell. Biol. 129:831-842.

33. Danilkovitch, A., Skeel, A., and Leonard, E.J. 1999. Macrophage stimulating protein-induced epithelial cell adhesion is mediated by a PI3-Kdependent, but FAK-independent mechanism. Exp. Cell Res. 248:575-582.

34. Xavier, R., Brennan, T., Li, Q., McCormack, C., and Seed, B. 1998. Membrane compartmentation is required for efficient $\mathrm{T}$ cell activation. Immunity. 8:723-732.

35. Stefanova, I., Horejsi, V., Ansotegui, I.J., Knapp, W., and Stockinger, H. 1991. GPI-anchored cell-surface molecules complexed to protein tyrosine kinases. Science. 254:1016-1019.

36. Nagel, W., et al. 1998. Phosphoinositide 3-OH kinase activates the beta2 integrin adhesion pathway and induces membrane recruitment of cytohesin-1. J. Biol. Chem. 273:14853-14861.

37. Adelsman, M.A., McCarthy, J.B., and Shimizu, Y. 1999. Stimulation of beta1-integrin function by epidermal growth factor and heregulin-beta has distinct requirements for erbB2 but a similar dependence on phosphoinositide 3-OH kinase. Mol. Biol. Cell. 10:2861-2878.

38. Satterthwaite, A.B., et al. 1998. Independent and opposing roles for Btk and lyn in B and myeloid signaling pathways. J. Exp. Med. 188:833-844.

39. Craxton, A., Jiang, A., Kurosaki, T., and Clark, E.A. 1999. Syk and Bruton's tyrosine kinase are required for B cell antigen receptor-mediated activation of the kinase Akt. J. Biol. Chem. 274:30644-30650.

40. Prenzel, N., et al. 1999. EGF receptor transactivation by G-protein-coupled receptors requires metalloproteinase cleavage of proHB-EGF. Nature. 402:884-888.

41. Eberl, L.P., Valdenaire, O., Saintgiorgio, V., Jeannin, J.F., and JuilleratJeanneret, L. 2000. Endothelin receptor blockade potentiates FasLinduced apoptosis in rat colon carcinoma cells. Int. J. Cancer. 86:182-187. 